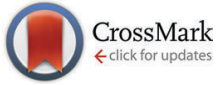

Cite this: Soft Matter, 2016, 12,4755

Received 17th February 2016, Accepted 11th April 2016

DOI: $10.1039 / c 6 s m 00412 a$

www.rsc.org/softmatter

\section{Nanometric thermal fluctuations of weakly confined biomembranes measured with microsecond time-resolution}

\author{
Cornelia Monzel, $\dagger^{\mathrm{ab}}$ Daniel Schmidt, ${ }^{\mathrm{cd}}$ Udo Seifert, ${ }^{\mathrm{c}}$ Ana-Sunčana Smith, ${ }^{\text {de }}$ \\ Rudolf Merkel $^{\mathrm{b}}$ and Kheya Sengupta*a
}

\begin{abstract}
We probe the bending fluctuations of bio-membranes using highly deflated giant unilamellar vesicles (GUVs) bound to a substrate by a weak potential arising from generic interactions. The substrate is either homogeneous, with GUVs bound only by the weak potential, or is chemically functionalized with a micro-pattern of very strong specific binders. In both cases, the weakly adhered membrane is seen to be confined at a well-defined distance above the surface while it continues to fluctuate strongly. We quantify the fluctuations of the weakly confined membrane at the substrate proximal surface as well as of the free membrane at the distal surface of the same GUV. This strategy enables us to probe in detail the damping of fluctuations in the presence of the substrate, and to independently measure the membrane tension and the strength of the generic interaction potential. Measurements were done using two complementary techniques - dynamic optical displacement spectroscopy (DODS, resolution: $20 \mathrm{~nm}, 10 \mu \mathrm{s}$ ), and dual wavelength reflection interference contrast microscopy (DW-RICM, resolution: $4 \mathrm{~nm}, 50 \mathrm{~ms}$ ). After accounting for the spatio-temporal resolution of the techniques, an excellent agreement between the two measurements was obtained. For both weakly confined systems we explore in detail the link between fluctuations on the one hand and membrane tension and the interaction potential on the other hand.
\end{abstract}

\section{Introduction}

In bio-membranes, spatial fluctuations, also called bending fluctuations, give rise to repulsive contributions and to intermembrane interactions. ${ }^{1-3}$ The physiological relevance of these fluctuations is highly debated, for example, in the case of erythrocyte membrane fluctuations and their ATP dependence. ${ }^{4-7}$

Thermally driven membrane fluctuations have been explored theoretically and experimentally since the $1970 \mathrm{~s},{ }^{1,3,8-10}$ starting with the pioneering studies by Helfrich ${ }^{1,3}$ who introduced the concept of steric repulsion of bilayer membranes calculated from their bending rigidity. These studies stimulated a comprehensive theoretical description of membrane fluctuations, ${ }^{11-16}$ accounting for the bilayer nature, ${ }^{17,18}$ membrane tension, ${ }^{19,20}$ molecular constituents ${ }^{21}$ as well as the interaction with a substrate..$^{22,23}$

\footnotetext{
${ }^{a}$ Aix-Marseille Université, CNRS UMR 7325 (Centre Interdisciplinaire de Nanosciences de Marseille - CINAM), Marseille Cedex 9, France. E-mail:sengupta@cinam.univ-mrs.fr, cornelia.monzel@curie.fr

${ }^{b}$ Institute of Complex Systems 7 (ICS-7), Forschungszentrum Jülich, Jülich, Germany

${ }^{c}$ II. Institut für Theoretische Physik, Universität Stuttgart, Germany

${ }^{d}$ Institut für Theoretische Physik, Friedrich Alexander Universität Erlangen-Nürnberg, Germany

${ }^{e}$ Division of Physical Chemistry, Ruđer Bošković Institute, 10000 Zagreb, Croatia

$\dagger$ Present address: Institut Curie, CNRS UMR 168, 75005 Paris Cedex 5, France.
}

To verify these concepts experimentally, membrane model systems were developed comprising solid supported lipid bilayers, membrane stacks, and giant unilamellar vesicles (GUVs) in a free, ${ }^{24-26}$ micropipette aspirated ${ }^{27}$ or adhered state. ${ }^{28-33}$ Most of these experimental studies focused on a precise evaluation of the membrane physico-chemical properties. ${ }^{34-36}$ Intriguingly, even for bending rigidity measurements, small-wavelength scattering experiments have identified anomalies, ${ }^{36-38}$ which may be associated with the extreme sensitivity of fluctuations to the surrounding buffer conditions. The use of mixed membranes may of course introduce micro- or nano-scale phase-separation, which in turn may impact the measured membrane properties. ${ }^{39}$

Fluctuations were also used to analyse membrane adhesion dynamics and equilibrium states. For the latter, an initial bond density regime of $\sim 10^{4} \mu \mathrm{m}^{-2}$ was mostly chosen, which largely exceeds the typical concentration of adhesion molecules on the cell surface of $\sim 10-100 \mu \mathrm{m}^{-2} .^{40}$ Adhesion in the limit of dilute bonds was investigated recently ${ }^{41-43}$ and gained much attention as adhesion structures similar to those of cells were observed. ${ }^{44}$ Still, data for weakly adhering membranes and the initial stages of adhesion are sparse and their properties are yet to be characterized in detail.

In addition, when studying the above models, a challenge arose whenever membrane properties were to be determined in 
the vicinity of a substrate: herein, membrane fluctuations are sterically restricted as the thermally excited out-of-plane fluctuations repel the membrane from the hard wall - an interaction usually referred to as Helfrich repulsion. Conversely, biological membranes near a wall may encounter attractive interactions, which, for the systems discussed herein, comprise van der Waals interaction and gravity. ${ }^{45}$ These generic interactions (likewise termed non-specific interactions) give rise to a total interaction potential of strength $\gamma$, which has the effect of rescaling membrane fluctuations. ${ }^{46,47}$ In the past, several experimental and data analysis strategies have been developed to determine the interaction potential $V(h)$. Yet, most of these strategies are case specific. For example, in the limit of strong adhesion, the classical contact angle equation by YoungDupré $^{48}$ can be used to determine the lateral membrane tension and the free energy of adhesion per unit area $W$, where $W \propto-V(h){ }^{19,30,49-52}$ Another strategy in the case of ultra weak gravity dominated confinement is to use the overall shape deformation of the GUV. ${ }^{52-54}$ Recently, we used the local equilibrium shape of the membrane to extract the detailed form of the interaction potential for the case of a membrane adhered to micropatterned substrates. ${ }^{55}$ In the present study, a general, case-independent strategy is presented where $\gamma$ is determined from the differences of fluctuations measured in the vicinity of the substrate and far away from it.

Finally, membrane fluctuations recorded with different techniques were hitherto difficult to compare since recordings are biased by the system resolution. Among the techniques predominately used to probe biomembranes shape fluctuations are flicker spectroscopy, ${ }^{24,56} \mathrm{X}$-ray, ${ }^{57,58}$ and neutron scattering. ${ }^{59,60}$ Close to a wall, the technique of choice has been reflection interference contrast microscopy (RICM), ${ }^{28,29,61-63}$ which is one of the techniques used in this work. RICM is limited to measurements in the substrate vicinity, due to the need for a reference beam for microinterferometry. Nevertheless, it is a label-free imaging technique which, in two wavelength implementation, probes membrane displacements in the normal direction with $4 \mathrm{~nm}$ resolution and with millisecond temporal precision. ${ }^{28,64}$ The second technique used in this work is dynamic optical displacement spectroscopy (DODS), ${ }^{7}$ which is complementary to RICM and based on a conventional fluorescence correlation spectroscopy (FCS) setup. ${ }^{65,66}$ It records physical displacements of a fluorescent membrane with $10 \mu$ s temporal and $20 \mathrm{~nm}$ spatial resolution. In comparison to camera based techniques like RICM this is a temporal resolution enhancement by two to three orders of magnitude. Moreover, DODS can measure at both the top, i.e., the distal surface of the GUV and in the vicinity of the substrate, i.e., the proximal surface. A comparable technique to DODS was recently introduced, ${ }^{67}$ which measures bending deformations in the lateral direction.

In order to compare data recorded using different techniques, we utilize an advanced theoretical framework which accounts for the spatio-temporal resolution of the setup. ${ }^{55}$ This permits the simultaneous discussion of RICM and DODS data in this work.

Using RICM and DODS we investigate membrane fluctuations in two GUV/substrate based model systems (see Fig. 1). In the first model system the GUV hovers above a flat homogeneous substrate and the substrate proximal membrane is confined by a weak, generic potential. We call this the "weakly confined" case. In the second model system, the GUV is adhered to a chemical pattern exhibiting regions of strong adhesion and regions of weak, generic adhesion only. Within weakly adhesive regions, the membrane may fluctuate similarly to the weakly confined case. We call this the "structured adhered" case. Both systems represent membranes close to the adhesion-deadhesion transition, ${ }^{68-70}$ as indicated by a circular shape of the GUV hemisphere. We trace membrane fluctuations over several orders of magnitude in space and time and, extract the tension, viscosity and the strength of the interaction potential from measurements at the distal and proximal surface. We propose an analysis protocol for compensating for finite resolution in experimental measurements, thus enabling us to

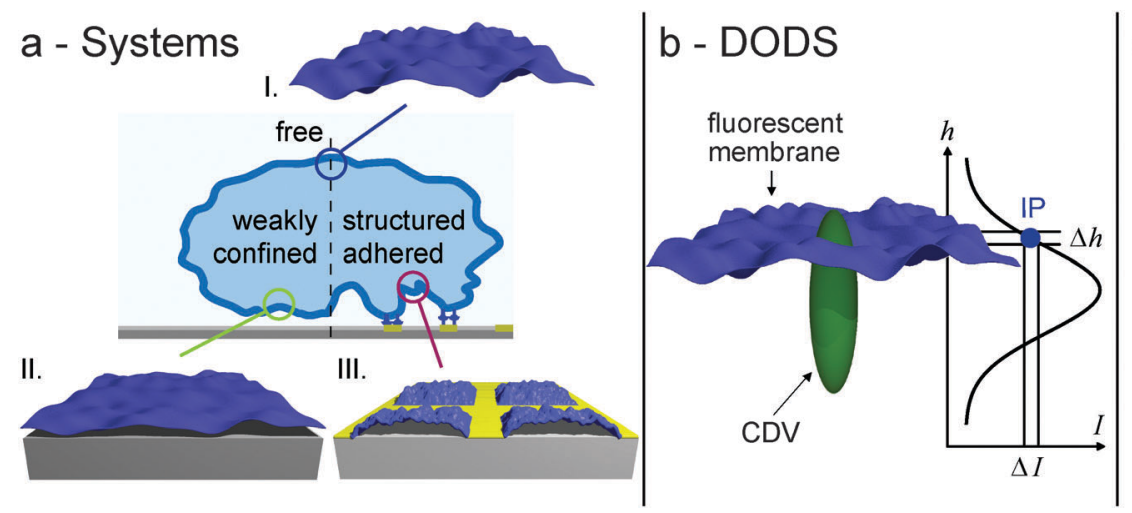

c - RICM

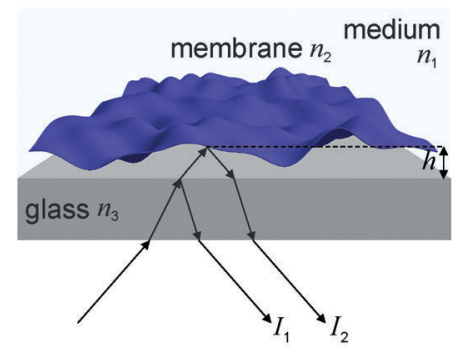

$I(x, t) \sim \cos \left(4 \pi n_{1} h(x, t) / \lambda\right)$

Fig. 1 (a) Sketch of systems investigated for nanometric membrane fluctuations: (I) free, unrestricted motion at the GUV distal side, (II) fluctuations of a weakly confined membrane near the substrate and (III) fluctuations of a structured adhered membrane. (b) Sketch of the DODS principle: a perpendicular intensity scan of the confocal detection volume (CDV) across the fluorescent membrane yields the inflection point (IP) of the intensity distribution. At this point physical displacements of the membrane, $\Delta h$, are most sensitively detected as intensity fluctuations, $\Delta l$. (c) Sketch of the RICM principle: incident light of wavelength $\lambda$ is reflected at interfaces of different refractive indices $n_{i}, i=1-3$, near the substrate. Reflected rays $\left(l_{1}, I_{2}\right)$ interfere with an intensity pattern $I(\mathbf{x}, t)$, from which the membrane height above the substrate, $h(\mathbf{x}, t)$, is determined. 
compare and combine RICM and DODS data in a meaningful way. In doing so, we achieve a comprehensive description of two weakly confined GUV systems. We find that in each case, the data are consistent with the Helfrich description, while pointing to an interaction potential that holds the membrane highly above the substrate.

\section{Experimental}

Materials

Lipids. SOPC (1-stearoyl-2-oleoyl-sn-glycero-3-phosphocholine), DOPE-PEG 2000 (1,2-dioleoyl-sn-glycero-3-phosphoethanolamine$N$-(methoxy(polyethyleneglycol)-2000)) and DOPE-cap-biotin (1,2-dioleoyl-sn-glycero-3-phosphoethanolamine- $N$-(cap biotinyl)) were purchased from Avanti Polar Lipids (Alabaster, AL, USA) and used without further purification. For DODS measurements, in addition, the fluorescently labelled lipid TRITC-DHPE ( $N$-(6-tetramethylrhodaminethiocarbamoyl)-1,2-dihexadecanoyl-sn-glycero3-phosphoethanolamine, triethylammonium salt) was purchased from Invitrogen (Eugene, OR, USA).

Proteins. Neutravidin covalently linked to the fluorescent label tetramethylrhodamine, henceforth called NAV-TMR, (Invitrogen) was reconstituted in PBS buffer $(140 \mathrm{mM} \mathrm{NaCl}$, $3 \mathrm{mM} \mathrm{KCl}, 10 \mathrm{mM} \mathrm{Na} \mathrm{HPO}_{4}, 2 \mathrm{mM} \mathrm{KH} \mathrm{KO}_{4}, \mathrm{pH} 7.2$ ) and ultracentrifuged at $137000 \mathrm{~g}$ and $4{ }^{\circ} \mathrm{C}$ for 2 hours to eliminate protein aggregates. Bovine serum albumin (BSA) and its covalently linked biotin conjugate (both: Sigma, Saint Louis, MO, USA) were dissolved in ultrapure water to a final concentration of $0.1 \mathrm{mg} \mathrm{mL}^{-1}$ and mixed in a $1: 1 \mathrm{w} / \mathrm{w}$ ratio (BSA-biotin mix).

Ultrapure water (Milli-Q Gradient A10, Millipore, San Francisco, CA, USA) was used throughout. Thickness corrected glass coverslips $(d=170 \pm 10 \mu \mathrm{m}$, Assistent, Karl Hecht KG, Sondheim, Germany) were cleaned by the following detergent treatment: ultrasonication in 2\% Hellmanex solution (Hellma, Müllheim, Germany) for 10 minutes, flushing thoroughly with ultrapure water and again ultrasonication $(2 \times 15$ minutes $)$ in ultrapure water followed by repeated flushing with the same.

\section{Substrate preparation}

Substrates with patterned receptor distribution were prepared by the micro-contact printing technique $(\mu \mathrm{CP}){ }^{71,72}$ For the preparation of microstamps see the detailed protocol published in Monzel et al. ${ }^{28}$ In brief, polyolefin plastomer (POP) stamps were obtained by hot embossing the POP onto a silicon wafer with the desired structure. An inking pad, made from a silicone elastomer mixed with a crosslinker (Sylgard 184, Dow Corning, MI, USA), was incubated with $400 \mu \mathrm{l}$ of the BSA-biotin mix, subsequently dried and used to ink the POP stamp. The latter was then pressed onto a cleaned coverslip thereby transferring the dried protein at the site of the pattern in a controlled manner. The patterned cover-slips, which served as substrates for GUV adhesion, were stored dry at $4-8{ }^{\circ} \mathrm{C}$ and used within 3 days. Prior to the experiment, the remaining regions of bare glass were passivated with $5 \mathrm{mg} \mathrm{mL}{ }^{-1}$ BSA for 15 minutes, the stamped region was functionalized with NAV-TMR by incubation for 30 minutes at a final concentration of $100 \mu \mathrm{g} \mathrm{mL}^{-1}$, and the passivation step with BSA was repeated. Homogeneous substrates for weakly confined GUV were prepared by passivating the glass with $5 \mathrm{mg} \mathrm{mL}^{-1}$ BSA for 15 minutes. In each case, excess protein was removed after each binding step by exchanging the buffer against protein free PBS.

\section{GUV preparation}

Giant unilamellar vesicles (GUVs) consisting of SOPC with $2 \mathrm{~mol} \%$ DOPE-PEG 2000 and 5 mol\% DOPE-cap-biotin (plus TRITC-DHPE at $1 \mathrm{~mol} \%$ for DODS experiments) were prepared via electro-swelling in 230 mOsm L $^{-1}$ sucrose (Sigma) solution. ${ }^{45,73}$ For experiments, vesicles were immersed in PBS buffer of $400 \mathrm{mOsm} \mathrm{L}^{-1}$ (187 mM NaCl, $4 \mathrm{mM} \mathrm{KCl,} 13 \mathrm{mM} \mathrm{Na} \mathrm{HPO}_{4}$, $2.7 \mathrm{mM} \mathrm{KH}_{2} \mathrm{PO}_{4}, \mathrm{pH} 7.2$ ) in a $1: 50$ dilution. The experimental chamber was then covered with a glass slide to avoid osmolarity changes due to evaporation. Measurements were started $30 \mathrm{~min}$ later to ensure full equilibration of the system. All measurements were carried out at room temperature.

\section{Data acquisition and analysis}

\section{DODS}

Previously, we used DODS to quantify membrane fluctuations in vesicles and cells. Herein, we summarize the basics of DODS which are relevant for the present study. A detailed description of the approach is given by Monzel et al. ${ }^{7}$ The basic idea of this method is to record intensity variations which originate from the fluctuation of a fluorescent membrane in a confocal detection volume (CDV, see Fig. 1b). Such a signature of membrane fluctuations is known to be visible in diffusion measurements with $\mathrm{FCS}^{74}$ but hitherto the focus was on evaluating the diffusion rather than fluctuations. Quantification of fluctuations, on the other hand, can be achieved with DODS by doping the membrane with fluorophores in a $1 \mathrm{~mol} \%$ concentration range whereby any signal fluctuation related to lipid diffusion is effectively suppressed. The following strategy then allows for an efficient detection of physical membrane displacements. First, an axial scan of the CDV at position $h_{\mathrm{CDV}}(t)$ across the membrane located at the mean position $\langle h\rangle$ is applied and the intensity along the way is recorded. The intensity can be approximated by a Gaussian function (see Fig. 1b)

$$
I\left(h_{\mathrm{CDV}}(t)\right)=I_{\max } \exp \left[-2 \frac{\left\{h_{\mathrm{CDV}}(t)-\langle h\rangle\right\}^{2}}{z_{0}^{2}}\right],
$$

where $I_{\max }$ denotes the maximally detected intensity and $z_{0}$ is the axial $1 / e^{2}$ - radius of the $\mathrm{CDV}^{7}$ In a second step, the membrane is positioned at the inflection point (IP), of the $\mathrm{CDV}$, given by $I\left(h_{\mathrm{CDV}}^{\mathrm{IP}}\right)=\mathrm{e}^{-1 / 2} I_{\max } \simeq 0.61 I_{\max }$. Here, the intensity gradient is maximal and, as a consequence, the sensitivity of fluctuation detection is the highest. This is the point where membrane displacements are recorded (Fig. 1b).

Fixing the CDV at the position of the IP, intensity fluctuations $\Delta I(h(t))$ arise from membrane fluctuations $\Delta h(t) \equiv$ $h(t)-\langle h\rangle$, with the instantaneous membrane position $h(t)$. 
Conversion from $\Delta I(h(t)) \rightarrow \Delta h(t)$ is realized via the slope $m$ at the IP, as measured from the axial intensity scan: ${ }^{7}$

$$
\Delta I(h(t)) \simeq m \Delta h(t) .
$$

In a similar manner, the intensity autocorrelation function $(\mathrm{ACF})$ is related to the membrane displacement autocorrelation function $(\mathrm{dACF} \equiv\langle\Delta h(\tau) \Delta h(0)\rangle)$ via

$$
\mathrm{ACF} \equiv \frac{\langle\Delta I(\tau) \Delta I(0)\rangle}{\langle I(\tau)\rangle^{2}}=\frac{m^{2}}{\langle I(\tau)\rangle^{2}} \mathrm{dACF} .
$$

Intensity fluctuations are recorded for a specific time interval, typically $2-3 \mathrm{~min}$, and the ACF is calculated.

DODS data were acquired with a FCS setup described in detail before: ${ }^{7}$ a laser scanning microscope (LSM710, Carl Zeiss, Göttingen, Germany) equipped with an FCS accessory (Confocor 3, Zeiss) and a 40× water immersion objective (C-Apochromat, NA 1.2, Zeiss) was used. The sample was illuminated with a $5 \mathrm{~mW}$ HeNe laser $(\lambda=543 \mathrm{~nm})$ and light was detected with 2 avalanche photodiodes (Perkin Elmer, Waltham, Ma, USA) for photon detection. The excitation laser was attenuated to a maximum count $I_{\max }$ of $70-100 \mathrm{kcps}$, which was well within the linear range of the detected fluorescence intensity. Appropriate filter sets were chosen (for the dye used here: beam splitter $488 / 543 \mathrm{~nm}$ for excitation and $580 \mathrm{~nm}$ long pass filter for emission, Zeiss) and the pinhole size was fixed to one Airy unit. Under these conditions the axial $1 / e^{2}$-radius $z_{0}$ amounts to $1284 \pm 11 \mathrm{~nm}(N=10)$. Errors reported throughout are standard deviations and indicated by \pm . Data acquisition and realtime autocorrelation were executed via the software ZEN (version 2008, Zeiss). Before the start of each DODS measurement, fluorescence and phase contrast images were acquired to determine the overall shape of the GUV and to choose the appropriate central position at the proximal or distal membrane.

\section{RICM}

For Dual Wavelength-RICM (DW-RICM) ${ }^{64}$ an inverted microscope (Axiovert200, Carl Zeiss) was equipped with an oil immersion objective (Antiflex EC Plan-Neofluar Ph3 63x/1.25, Zeiss) containing a $\lambda / 4$ plate. Light emitted by a metal halogenide lamp (X-Cite, Exfo, Quebec, Canada) was filtered using a dualband interference filter $\left(\lambda_{\mathrm{g}}=546 \pm 10 \mathrm{~nm}\right.$ and $\lambda_{\mathrm{b}}=436 \pm$ $20 \mathrm{~nm}$ ). The numerical aperture of illumination was set to 0.54 . In order to achieve maximum contrast the antiflex technique was applied. ${ }^{75}$ Two micrographs were recorded simultaneously. For this purpose, the reflected light was split according to its wavelength (FT $460 \mathrm{~nm}, \mathrm{LP} 470 \mathrm{~nm}$ (Zeiss) and BP $436 \pm 10 \mathrm{~nm}$ (AHF, Tübingen, Germany)) and focused on two separate digital CCD cameras where one was triggered by the other (sensicam qe, PCO, Kehlheim, Germany). Image recording was controlled by the software OpenBox (version 1.77, Informationssysteme Schilling, Munich, Germany). 2000 consecutive micrographs with a frame rate of $20 \mathrm{~Hz}$ were recorded. Analysis was performed considering the interfaces of glass/outer buffer, outer buffer/membrane and membrane/inner buffer in the analysis within the square regions of the fluctuating membrane (for details see Monzel et $a l^{28}{ }^{28}$. Data were analyzed using self-written routines in Matlab (version 3.0 (R2010b), The MathWorks, Inc. MA, USA) utilizing the image processing toolbox and ImageJ (version 1.45s, Rasband, W.S., NIH, Bethesda, MD, USA).

Knowing the refractive indices and thicknesses of the different layers and using Fresnel's equations the detected intensity $I(h)$ is calculated. The theory of partial coherent light states that these data follow the intensity-height relation ${ }^{52}$

$$
I(h)=\frac{S}{2}-D \frac{\sin (y)}{2 y} \cos \left(2 k n_{1}\left[h \cos ^{2}\left(\frac{\alpha}{2}\right)-h_{\text {off }}\right]\right)
$$

and a fit with this equation gives the absolute membrane heights $h=h(\mathbf{x}, t) \cdot y=2 k h \sin ^{2}\left(\frac{\alpha}{2}\right)$ with the half angle $\alpha=24^{\circ}$ of the cone of illumination being used to account for the illumination numerical aperture. The offset $h_{\text {off }}=35 \mathrm{~nm}^{76}$ and $S$ and $D$, the sum and difference of the maximal and minimal intensity, respectively, are determined from the fit. $k=2 \pi / \lambda$ is the wave vector and $n_{1}=1.335$ the refractive index of the outer buffer. The phase ambiguity arising from the cosine in eqn (4) is lifted using DW-RICM instead of conventional RICM. Here, two interferograms for wavelengths $\lambda=546 \mathrm{~nm}$ and $436 \mathrm{~nm}$ are recorded simultaneously and from the twofold intensity information a unique height of the object above the substrate can be derived. ${ }^{64}$ A detailed description of this procedure can be found elsewhere. ${ }^{28}$

\section{Correcting for finite resolution}

Since the detected, apparent membrane heights $\bar{h}(\mathbf{x}, t)$ are always spatio-temporal averages of the instantaneous height $h(\mathbf{x}, t)$, the detected apparent fluctuations $\Delta \bar{h}(\mathbf{x}, t)$ and the dACF $\langle\Delta \bar{h}(\mathbf{x}, t) \Delta \bar{h}(\mathbf{x}, 0)\rangle$ were corrected to account for the resolution of the setup. In the following we describe the theoretical context relevant for this work and account for (i) the spatio-temporal resolution of the setup, (ii) the detection limits of the system, and (iii) the boundary conditions of the investigated membranes (see Fig. 1a).

In all cases, the correct form of the $\mathrm{AACF}$ is derived starting from the classical theory for membrane height fluctuations, which describes the membrane as a two-dimensional sheet with bending rigidity $\kappa$ and membrane tension $\sigma$. In the vicinity of a wall an additional term accounts for the interaction potential $V(h)$ which in harmonic approximation has a minimum at $h_{0}$ and a curvature $\gamma$. The Hamiltonian is given by

$$
\mathcal{H}=\int_{\mathcal{S}} \mathrm{d} \mathbf{x}\left[\frac{\kappa}{2}\left(\nabla^{2} h\right)^{2}+\frac{\sigma}{2}(\nabla h)^{2}+\frac{\gamma}{2}\left(h-h_{0}\right)^{2}\right]
$$

where $h=h(\mathbf{x})$, with $\mathbf{x} \equiv(x, y)$ the lateral position on the membrane, and $h_{0} \equiv\langle h(\mathbf{x})\rangle$ in equilibrium. Far from the substrate at the distal membrane $\gamma \equiv 0$.

The true dACF without finite resolution effects is given by ${ }^{14}$

$$
\langle\Delta h(\mathbf{x}, 0) \Delta h(\mathbf{x}, \tau)\rangle=\frac{k_{\mathrm{B}} T}{(2 \pi)^{2}} \int \mathrm{d} q \frac{\mathrm{e}^{-\Gamma(q) \tau}}{\kappa q^{3}+\sigma q+\gamma / q} .
$$


At $\tau \equiv 0$ the dACF yields the root mean square fluctuation amplitude $\psi=\sqrt{\left\langle\Delta h(\mathbf{x}, 0)^{2}\right\rangle}$. The coefficient $\Gamma(q)$ accounts for the hydrodynamic damping and relaxation of membrane fluctuations. At the distal membrane it takes the well known form $\Gamma(q)=\left(\kappa q^{4}+\sigma q^{2}\right) /(4 \eta q) .{ }^{14}$ For the proximal membrane $\Gamma(q)$ is somewhat enhanced ${ }^{55,77}$

$$
\begin{aligned}
\Gamma(q)= & \frac{\left(\kappa q^{4}+\sigma q^{2}+\gamma\right)}{4 \eta q} \\
& \times \frac{2\left(\sinh ^{2}(q h)-(q h)^{2}\right)}{\sinh ^{2}(q h)-(q h)^{2}+\sinh (q h) \cosh (q h)+(q h)} .
\end{aligned}
$$

The bending rigidity for SOPC lipid membranes, used throughout here, is $\kappa=20 k_{\mathrm{B}} T .^{78}$ The viscosity $\eta$, is calculated as the arithmetic mean of the viscosity of the inner GUV buffer and outer buffer (see Appendix 1).

DODS. In the case of DODS the detection system is an avalanche photodiode which counts the photons originating from the fluorophores within the CDV. The CDV in turn has an intrinsic intensity distribution and only the integral value of intensities in the cross-section between the CDV and membrane are detected. Consequently, the true membrane fluctuations $\Delta h(\mathbf{x}, t)$ are smeared out to the apparent fluctuations $\Delta \bar{h}(\mathbf{x}, t)$, and these emerge from a convolution of the lateral point spread function $P(\mathbf{x})=2 /\left(\pi \omega^{2}\right) \exp \left(-2 \mathbf{x}^{2} / \omega^{2}\right)$ at $h(\mathbf{x}, t)$ and the fluctuations $\Delta h(\mathbf{x}, t)$. Note that, due to measurements at the IP the lateral $1 / e^{2}$-radius $\omega_{0}$ of the point spread function $P(\mathbf{x})$ increases to $^{79}$

$$
\omega^{2}=\omega_{0}^{2}\left(1+\frac{\lambda^{2}\left(h_{\mathrm{CDV}}^{\mathrm{IP}}-\langle h\rangle\right)^{2}}{\pi^{2} n_{1}^{2} \omega_{0}^{4}}\right)
$$

$n_{1}$ is the refractive index of the bulk medium and $\lambda$ the wavelength of the excitation light. For our measurement conditions $\omega_{0}=281 \pm 7 \mathrm{~nm}(N=10$; standard deviation error $)$. With these considerations and integrating over $\mathbf{x}$, the dACF in case of DODS reads

$$
\begin{aligned}
\langle\Delta \bar{h}(0) \Delta \bar{h}(\tau)\rangle^{\text {DODS }} & =\int_{0}^{\vartheta} \int_{0}^{\vartheta} \frac{\mathrm{d} t_{1}{ }^{\prime} \mathrm{d} t_{2}^{\prime}}{\vartheta^{2}}\left\langle\Delta \bar{h}\left(t_{1}^{\prime}\right) \Delta \bar{h}\left(\tau+t_{2}^{\prime}\right)\right\rangle \text { DODS } \\
& =\frac{k_{\mathrm{B}} T}{2 \pi} \int_{q_{\min }}^{\infty} \mathrm{d} q \frac{q \mathrm{e}^{-\Gamma(q) \tau}}{\kappa q^{4}+\sigma q^{2}+\gamma} \mathrm{e}^{-\frac{1}{4} \omega^{2} q^{2}} \psi_{\vartheta}(q),
\end{aligned}
$$

where the averaged time component is given by

$$
\psi_{\vartheta}(q)=\frac{\mathrm{e}^{-\Gamma(q) \vartheta}-1+\Gamma(q) \vartheta}{\Gamma^{2}(q) \vartheta^{2}} .
$$

In comparison to the classical expression, the spatial averaging due to finite resolution only contributes an additional factor $\phi^{\text {DODS }}(q)=\exp \left(-\omega^{2} q^{2} / 4\right)$ see eqn (8). This factor dampens the detection of high modes at the upper integral limit $q_{\max }=\infty$. At low frequencies the accessible modes are limited due to the finite size of the vesicle, i.e., the wavelength spanning the whole vesicle $q_{\min }=\sqrt{3} / R$ (see Appendix 2). Note that in the limit of $\kappa$ dominated fluctuations $\kappa q^{2} \gg \sigma$, and in the limit of $\sigma$ dominated
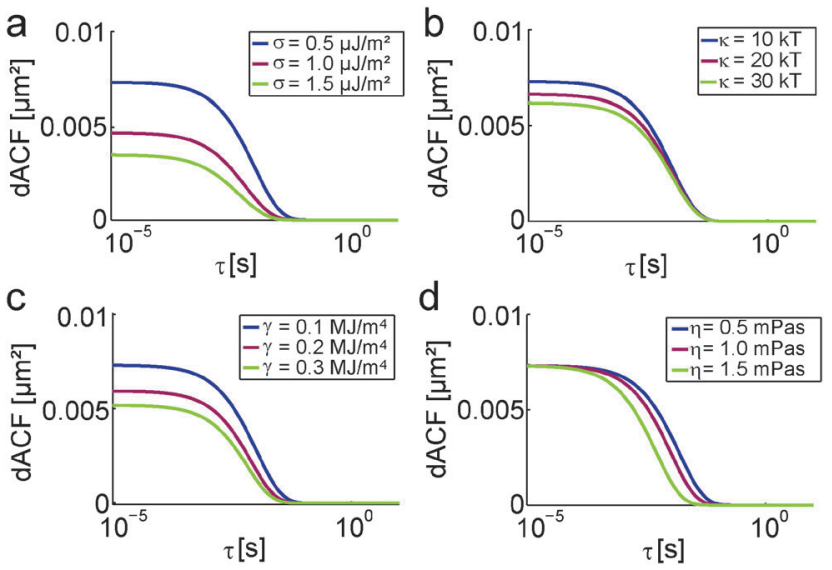

Fig. 2 Theoretical dACF variation with membrane/system parameters (eqn (8)): (a) membrane tension $\sigma$, (b) bending rigidity $\kappa$, (c) interaction potential strength $\gamma$, and (d) effective viscosity $\eta$. Default values are $\sigma=0.5 \mu \mathrm{J} \mathrm{m}^{-2}$, $\gamma=0.1 \mathrm{MJ} \mathrm{m}^{-4}, \kappa=10 \mathrm{k}_{\mathrm{B}} T, \eta=1.0 \mathrm{mPa} \mathrm{s}$.

fluctuations $\kappa q^{2} \ll \sigma . \sigma$ is typically of the order of $0.01-1 \mu \mathrm{J} \mathrm{m}^{-2}$ and $\kappa q^{2}$ runs from $\sim 10^{-5}-1 \mu \mathrm{J} \mathrm{m}^{-2}$. Above the latter value the integrand is highly damped, wherefore small $q$ values dominate the integral and $\sigma>\kappa q^{2}$ holds for the majority of investigated objects. Thus, in our case DODS is most sensitive to $\sigma$. A similar argument can be used to explain the sensitivity for $\gamma$.

Using eqn (8), the theoretical shape of the dACF in case of DODS was calculated for typical system parameters and the results are illustrated in Fig. 2. The shape of the dACF is strongly influenced by changes in $\sigma$ and somewhat less in $\gamma$, while $\kappa$ and $\eta$ have only little effect on the amplitude and relaxation of the function, respectively.

RICM. In case of RICM, true fluctuations are blurred to the apparent fluctuations in a similar manner. The camera based detection results in spatial averaging of the intensity over the, ideally resolution matched, pixel size $A$. The camera exposure time $\vartheta$ restricts the detected frequencies to values $<1 /(2 \vartheta)$. Consequently, the apparent heights $\bar{h}(\mathbf{x}, t)$ follow

$$
\bar{h}(\mathbf{x}, t)=\int_{0}^{\vartheta} \frac{\mathrm{d} t^{\prime}}{\vartheta} \int_{A} \frac{\mathrm{d} \mathbf{x}^{\prime}}{A} h\left(\mathbf{x}+\mathbf{x}^{\prime}, t+t^{\prime}\right) .
$$

The apparent autocorrelation function emerges - similar to DODS - from a convolution of the true correlations (using eqn (6)) with the effects of the spatio-temporal averaging ${ }^{55}$

$$
\langle\Delta \bar{h}(\mathbf{x}, 0) \Delta \bar{h}(\mathbf{x}, \tau)\rangle^{\mathrm{RICM}}=\frac{k_{\mathrm{B}} T}{(2 \pi)^{2}} \int \mathrm{d} \mathbf{q} \frac{\mathrm{e}^{-\Gamma(q) \tau}}{\kappa q^{4}+\sigma q^{2}+\gamma} \phi^{\mathrm{RICM}}(\mathbf{q}) \psi_{\vartheta}(\mathbf{q}),
$$

where $\phi^{\mathrm{RICM}}(\mathbf{q})$ is a function of the spatial component

$$
\phi^{\mathrm{RICM}}(\mathbf{q})=\iint_{A} \frac{\mathrm{d} \mathbf{x}_{1}{ }^{\prime} \mathrm{d} \mathbf{x}_{2}{ }^{\prime}}{A^{2}} \mathrm{e}^{-i q\left(\mathbf{x}_{1}{ }^{\prime}-\mathbf{x}_{2}{ }^{\prime}\right)} .
$$

$\psi_{9}(\mathbf{q})$ accounts for the temporal averaging as given before.

The effect of finite resolution on the apparent mean square fluctuations $\left\langle\Delta \bar{h}^{2}\right\rangle$ and their dependence on $\sigma$ are illustrated in Fig. 3. For RICM compared to DODS $\left\langle\Delta \bar{h}^{2}\right\rangle$ increases by more than a factor of 5 (see Fig. 3a). Thus, accounting for finite 

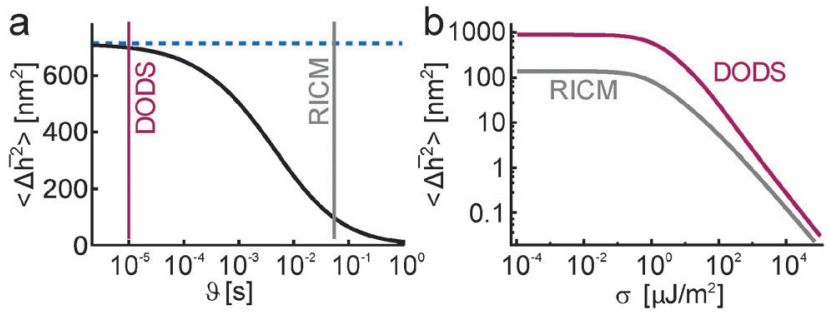

Fig. 3 (a) Calculated effect of temporal resolution $\vartheta$ on fluctuation amplitude $\left\langle\Delta \bar{h}^{2}\right\rangle$ for a weakly confined GUV at typical parameters $\sigma=$ $0.5 \mu \mathrm{J} \mathrm{m}^{-2}, \gamma=4 \mathrm{MJ} \mathrm{m}^{-4}$ and $h_{0}=600 \mathrm{~nm}$ (black line). Vertical lines indicate DODS and RICM temporal resolution, dashed line denotes infinite resolution. (b) $\left\langle\Delta \bar{h}^{2}\right\rangle$ calculated as a function of membrane tension $\sigma$, for the spatio-temporal resolution of RICM and DODS.

resolution enables us to correctly rescale the measured fluctuations, and permits quantitative comparison between different techniques.

Throughout, for both DODS and RICM, we correct the apparent fluctuations $\sqrt{\left\langle\Delta \bar{h}^{2}\right\rangle}$ and correlation functions $\langle\Delta \bar{h}(0) \Delta \bar{h}(\tau)\rangle$ to the instantaneous fluctuations $\psi=\sqrt{\left\langle\Delta h^{2}\right\rangle}$ and correlation functions $\langle\Delta h(0) \Delta h(\tau)\rangle$. Unless otherwise stated, all reported values are already corrected.

\section{Results and discussion}

\section{Weakly confined membranes}

In the experiment GUVs sedimented and hovered over the substrate, which was passivated to prevent strong attraction. The proximal membrane within the vesicle substrate contact zone was on average flat. Still, the membrane was highly fluctuating, indicating that it was confined in a generic potential. For measurements, vesicles with radii $\geq 10 \mu \mathrm{m}$ were chosen to ensure membrane flatness within the detection volume. $\$$ When the vesicle has equilibrated to ambient conditions and no lateral drift on the timescales of the measurement ( $\simeq 5 \mathrm{~min}$ ) was detectable, the CDV was adjusted such that the membrane was vertically positioned at the IP before intensity fluctuations were recorded (see Fig. 4a).

First, we measured fluctuations at the distal surface of the GUV using DODS. Data were collected from the centre of the distal membrane of the vesicle. From these data the dACF was calculated using eqn (3). Fluctuation amplitudes were determined from the intercept of the dACF (i.e., its value at $\tau \equiv 10^{-5} \mathrm{~s}$ ), yielding an average value of $\psi_{\text {distal }}^{\text {DODS }}=67 \pm 19 \mathrm{~nm}(N=20$; standard deviation error). Values of $\psi_{\text {distal }} \simeq 100 \mathrm{~nm}$ are expected for GUVs that are freely fluctuating far from any surface. However, due to the lack of suitable techniques these were so far only measured using flicker spectroscopy at a spatio-temporal resolution of $30 \mathrm{~Hz}$ and $30 \mathrm{~nm}^{24,56,80,81}$

Next, DODS data were collected at the proximal membrane of the vesicle. Here, membrane fluctuations are damped due to

\$ For vesicle radii $\geq 10 \mu \mathrm{m}$ the spherical membrane shape deviates by less than $0.3 \mathrm{~nm}$ from a plane within the CDV.
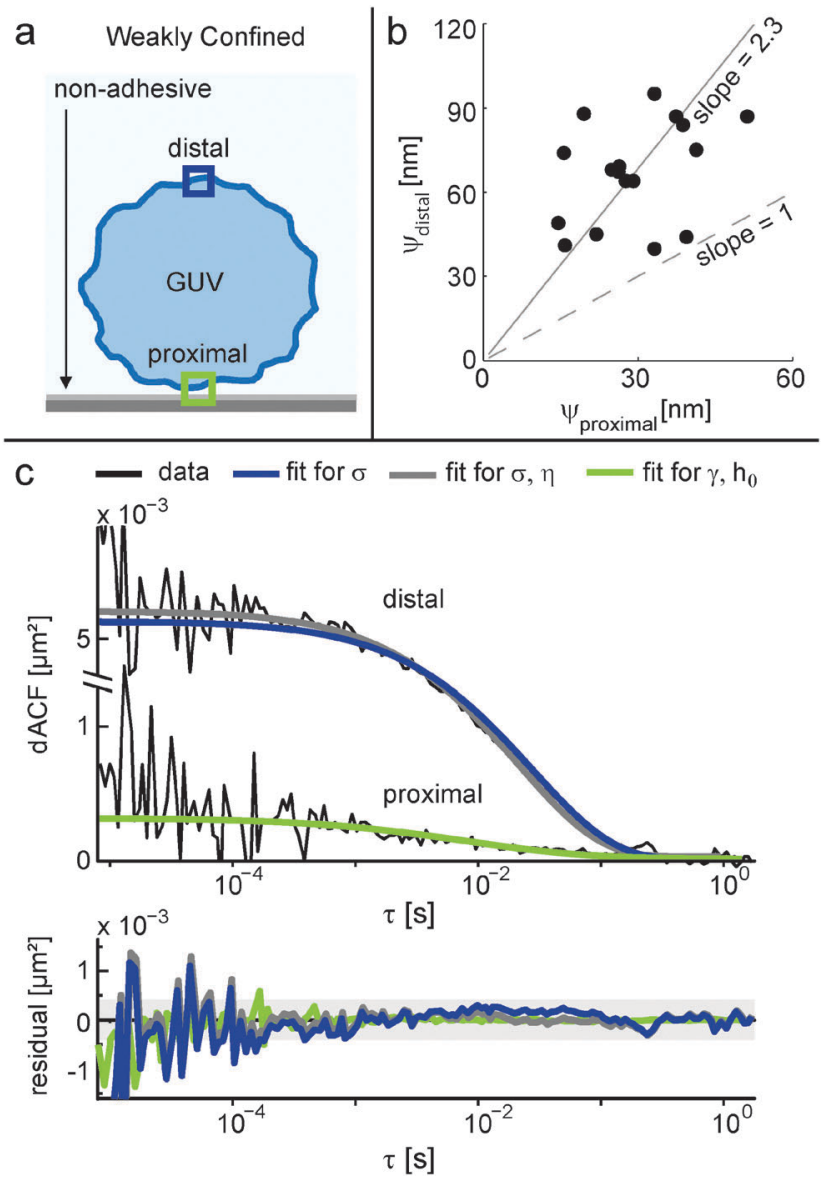

Fig. 4 GUV confined by a weak generic potential. (a) Sketch of the system. Colored boxes indicate measurement positions at distal (blue) and proximal (green) surfaces. (b) Relation between fluctuation amplitudes $\psi$ at the distal and the proximal surface of each vesicle. Fluctuations at the distal surface are on average enhanced by a factor $\times 2.3$ (solid line). (c) Typical dACFs. $\sigma$ is obtained from a single parameter fit to the dACF at the distal surface (blue). A two parameter fit for $\sigma$ and $\eta$ (grey) is plotted for comparison. $\gamma$ and $h_{0}$ are determined from a fit of the dACF at the proximal surface (green). Residuals remain mostly below the resolution limit (grey bar).

the presence of the substrate and consequently the average amplitude reduced to $\psi_{\text {proximal }}^{\text {DODS }}=29 \pm 10 \mathrm{~nm}(N=17)$. This value can be compared to previous studies on weakly adhering vesicles, where amplitudes of 9 to $34 \mathrm{~nm}$ were detected using RICM (here we corrected the reported values to account for the spatio-temporal averaging inherent to RICM). ${ }^{29,30,42}$ Note that in this study GUVs were prepared in such a way that they had higher osmotic deflation (osmotic difference: 170 mOsm L $\mathrm{L}^{-1}$ ) as compared to the studies cited here (20-100 mOsm L $\left.{ }^{-1}\right)$.

Measurements at both distal and proximal surfaces of the same vesicle enable the direct assessment of the influence of the substrate on the fluctuation amplitude. Since the membrane tension is the same everywhere in the vesicle, changes in membrane fluctuation near the substrate directly reflect the changes due to the membrane-substrate interaction. Fig. $4 \mathrm{~b}$ illustrates the attenuation of fluctuations from the distal to the proximal membrane, which on average amounts to $\times 2.3$. 
From the measurement at the distal membrane, and assuming a bending rigidity of $\kappa=20 k_{\mathrm{B}} T,{ }^{78}$ the membrane tension can be uniquely determined based on the mean square fluctuation amplitude (eqn (8) with $\tau$ and $\gamma \equiv 0$ ). Alternatively, it can be determined from the full ACF. Its decay is given by the classical expression for the damping coefficient $\Gamma(q)$, where in addition to $\sigma$, the buffer viscosity $\eta$ is used as a fit parameter. The resulting fit shows convincing agreement with data and for correlation times $\tau>10^{-4} \mathrm{~s}$ the residuals remain below the resolution limit (see Fig. 4c).

The membrane tension was found to be between $0.2-1.4 \mu \mathrm{J} \mathrm{m}^{-2}$ and the average value of the viscosity was $\eta=1.2 \pm 0.6 \mathrm{mPa} s$ $(N=20$; standard deviation error). The latter accords with the expected value of $\eta=1.2 \mathrm{mPa}$, which is the arithmetic mean of the outer buffer viscosity $\eta=1.0 \mathrm{mPa} s$ and the viscosity of the sucrose solution inside the vesicle $\eta=1.4 \mathrm{mPa}$ s (see Appendix 2). The same viscosity was also measured using FCS by Cha et al. ${ }^{82}$ in a similar model system.

Having established that the viscosity is indeed as expected, we fixed its value to $\eta=1.2 \mathrm{mPa} \mathrm{s}$ and performed a one parameter fit with the tension as the only free parameter. Correspondence between data and the one-parameter fit remained high (see residuals in Fig. 4c) and changes in membrane tension values between two-parameter fit $(\sigma=0.52 \pm$ $\left.0.39 \mu \mathrm{J} \mathrm{m}^{-2}\right)$ and one-parameter fit $\left(0.51 \pm 0.37 \mu \mathrm{J} \mathrm{m}^{-2}\right)$ were negligibly small. This analysis demonstrates the robustness of the determination of $\sigma$ using this technique. Note that the observed variation in $\sigma$ resulted from real tension variations between different GUVs. To test the influence of measurement uncertainty we undertook repeated DODS measurements on the same vesicle (Table 1). Performing one parameter fits the membrane tension was determined to an accuracy of $\pm 0.035 \mu \mathrm{J} \mathrm{m}^{-2}$.

Absolute tension values are in excellent agreement with results estimated from other fluctuation dominated systems, e.g., unbound GUVs analyzed with flicker spectroscopy ${ }^{24,56}$ and neutron scattering ${ }^{83}$ or weakly adhered vesicles analyzed using RICM $^{30,84}$ where tensions were of the order of $\sim 0.1 \mu \mathrm{J} \mathrm{m}^{-2}$. In more tightly adhered or micropipette aspirated GUVs membrane tensions are considerably larger and of the order of $\sim 1 \mu \mathrm{J} \mathrm{m}^{-2}$ and $\sim 1 \mathrm{~mJ} \mathrm{~m}^{-2}$, respectively. ${ }^{29,67}$

The dACFs of the proximal membrane were fitted using eqn (7) and (8), with fitting parameters $\gamma$ and $h_{0}$, the latter being the equilibrium height above the substrate. $\sigma$ was fixed to the value determined from the one-parameter fit at the distal side of the same vesicle. A typical example is depicted in Fig. 4c. The fit yielded high correspondence with data and residuals remained below the resolution limit for $\tau>10^{-4} \mathrm{~s}$, as before. Hence, this is the first time that $\sigma$ and $\gamma$ could be independently measured in direct experiments.

Table 1 Tension $\sigma$ (in $\mu \mathrm{J} \mathrm{m}^{-2}$ ) for two exemplary GUVs, (GUV1 and GUV2) and for repeated DODS measurements $M 1, M 2$ and $M 3$ of the same vesicle

\begin{tabular}{llll}
\hline & M1 & M2 & M3 \\
\hline$\sigma$ of GUV1 & 0.41 & 0.46 & 0.48 \\
$\sigma$ of GUV2 & 0.36 & 0.32 & 0.37
\end{tabular}

The interaction potential strength $\gamma$ was between $0.7-20 \times$ $10^{6} \mathrm{~J} \mathrm{~m}^{-4}$ and $h_{0}$ between 150 and $654 \mathrm{~nm}$. For the latter, the higher variation in the case of DODS arises from the limited sensitivity of this technique for $h_{0}$. The absolute values of $\gamma$ were consistent with previously reported results for similar systems as measured with RICM. ${ }^{29,30,85}$

\section{Structured adhered vesicles}

GUVs were allowed to sediment and interact with the substrates bearing the adhesive grid patterns. They adhered to the substrate in a way that part of the proximal membrane became tightly bound while square-shaped patches $\left(4 \times 4 \mu^{2}\right)$ remained free to fluctuate (see Fig. 5a). The selective pinning of the membrane to the substrate yields substantial control over absolute membrane heights and renders the system particularly amenable for RICM analyses. In our previous study we investigated similar systems with DW-RICM and demonstrated that the lipids on the membrane are free to diffuse across the adhered regions. ${ }^{45}$ Therefore, the membrane within each square is expected to reach an equilibrium state.

DODS measurements were performed at the distal, and the fluctuating parts of the proximal membrane. Fig. $5 \mathrm{~b}$ illustrates

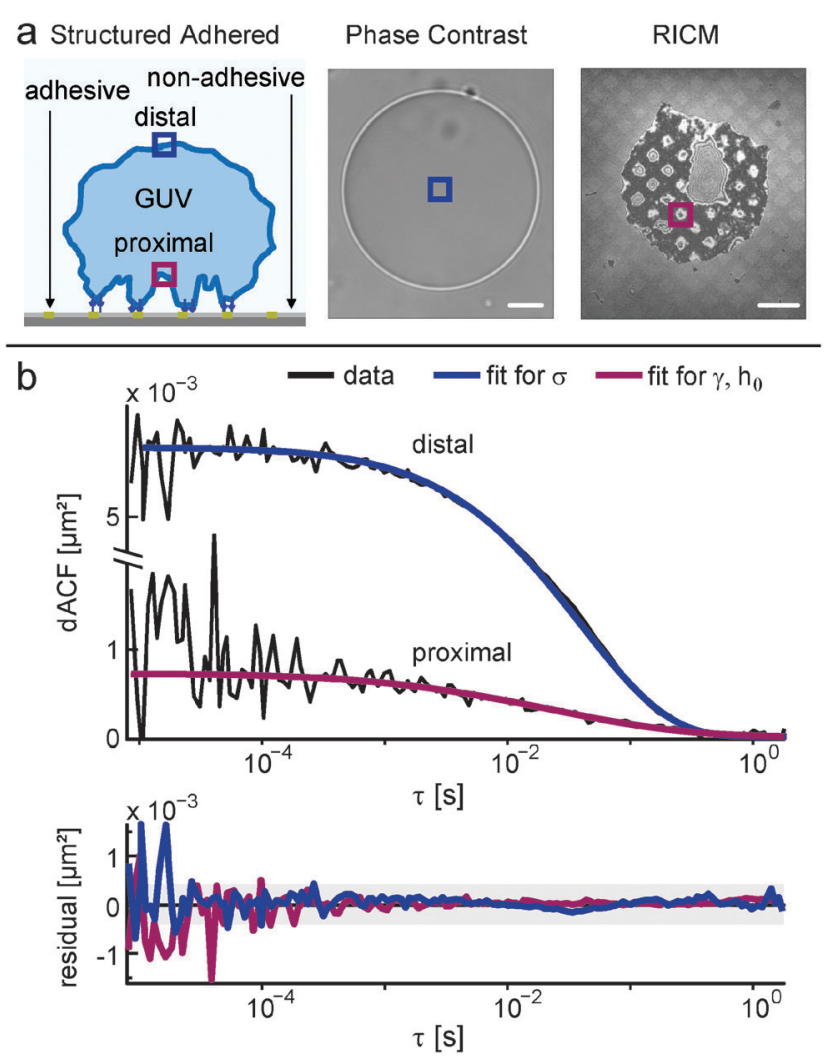

Fig. 5 Structured adhered GUV. (a) Sketch of the system (left), the phase contrast image of the vesicle (middle) and the RICM image of the vesiclesubstrate contact zone (right). Colored boxes indicate measurement positions at the vesicle distal side and within fluctuation areas at the proximal side. Scale bar $10 \mu \mathrm{m}$. (b) Typical dACFs. $\sigma$ is obtained from a single parameter fit to the dACF at the vesicle distal side (blue). $\gamma$ and $h_{0}$ are determined from a fit to the $\mathrm{dACF}$ at the proximal side (purple). Residuals remain mostly below the resolution limit (grey area). 
Table 2 DODS and RICM results: average and standard deviation of fluctuation amplitude, $\psi_{\text {proximal, }}$ and membrane height, $h_{0}(N=14$ for DODS and RICM data)

\begin{tabular}{lll}
\hline Technique & $\psi_{\text {proximal }}[\mathrm{nm}]$ & $h_{0}[\mathrm{~nm}]$ \\
\hline DODS & $31 \pm 12$ & $402 \pm 252$ \\
RICM & $26 \pm 21$ & $538 \pm 56$
\end{tabular}

a typical example of dACF measurements, along with the singleparameter fit for $\sigma$ at the distal membrane and the simultaneous fit for $\gamma$ and $h_{0}$ at the proximal membrane. Both fits yielded high correspondence with data and residuals remaining below the resolution limit for correlation times $\tau>3 \times$ $10^{-5} \mathrm{~s}$ (grey area in Fig. 5b). The fluctuation amplitude at the distal membrane amounts to $\psi_{\text {distal }}^{\mathrm{DODS}}=82 \pm 23 \mathrm{~nm}(N=16)$ and is enhanced by a factor $\times 2.7$ compared to their amplitudes measured at the proximal membrane. This is a similar enhancement as obtained for the weakly confined system. Parameters $\psi_{\text {proximal }}, \sigma, \gamma$, and $h_{0}$ as obtained from the fits are explicitly given in Tables 2 and 3, and discussed as follows.

For the structured adhered system RICM measurements at the proximal membrane were performed and compared to DODS data utilizing the theory accounting for finite resolution (see Fig. 3a). Results of both measurements are given in Table 2. Note that DODS is more suited for the determination of $\psi_{\text {proximal }}$ whereas RICM yields better results for $h_{0}$. Both of these values, $\psi_{\text {proximal }}^{\mathrm{DODS}}$ and $h_{0}^{\mathrm{RICM}}$, are slightly higher than their values obtained using other techniques but assessing the deviation via the Wilcoxon rank sum test yielded no statistical difference between data sets (significance level $p=0.27$ for $\psi_{\text {proximal }}$, and $p=0.23$ for $h_{0}$ ).

The standard deviation given in Table 2 represents the true distribution of fluctuation amplitudes GUVs exhibit. It cannot be solely attributed to measurement inaccuracy. This is different in the case of $h_{0}$ measured using DODS. Here, the large standard deviation compared to DW-RICM results from the lower sensitivity of DODS for $h_{0}$. Thus, for optimal results $h_{0}$ should be determined via RICM and $\sigma$ via DODS measurements. So far we could not perform DODS and DW-RICM on the same setup and the same GUV, wherefore in the following we compare the average values over several GUVs.

Previous work on confined adhesion to substrate patterns used vesicles that were tenser than ours and reported fluctuation amplitudes of $\psi_{\text {proximal }}=9-37 \mathrm{~nm}^{29,30}$ (corrected for finite resolution) as well as equilibrium heights of $h_{0}=31-49 \mathrm{~nm}^{29}$ Clearly, the differences to the present work arise from different membrane tension.

In the following text we present the results for the weakly confined and structured adhered system and compare them to
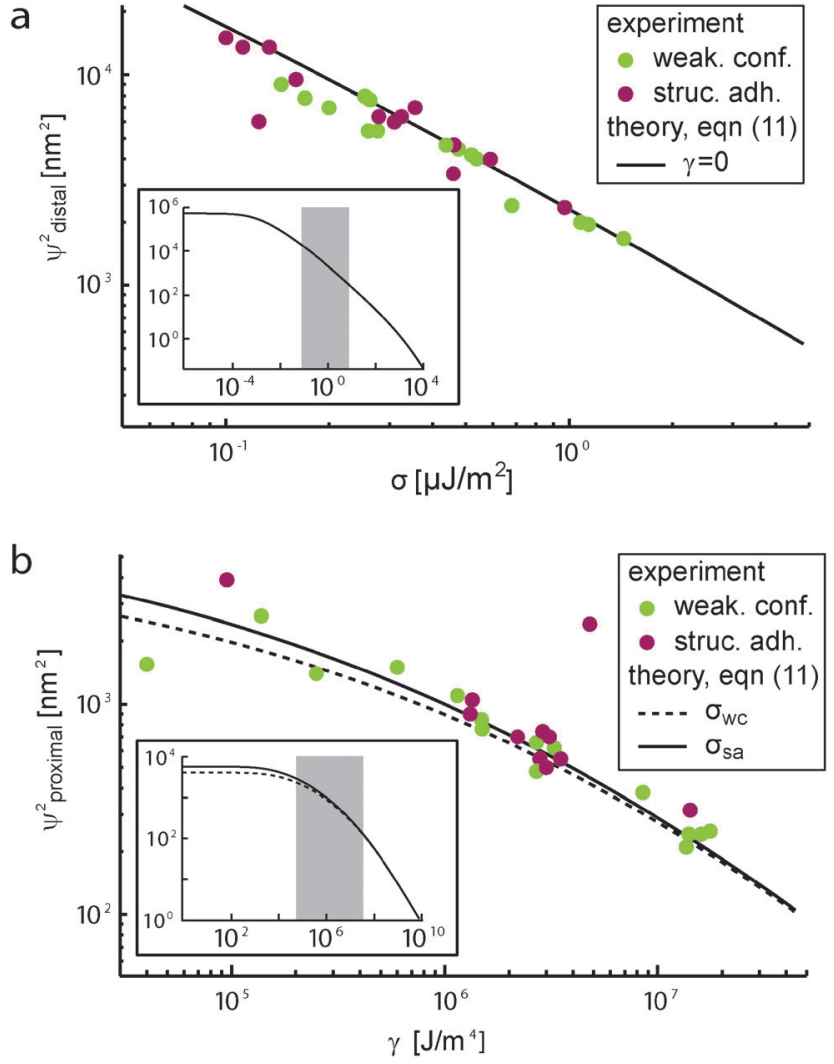

Fig. 6 Relation between fluctuation amplitude and membrane/system parameters: (a) $\psi_{\text {distal }}{ }^{2}$ ( $\left.\equiv\left\langle\Delta h^{2}\right\rangle_{\text {distal }}\right)$ vs. $\sigma$ for weakly confined (green) and structured adhered values (purple). Data follow the theory, eqn (8). (b) $\psi_{\text {proximal }}{ }^{2}$ ( $\left.\equiv\left\langle\Delta h^{2}\right\rangle_{\text {proximal }}\right)$ vs. $\gamma$ for weakly confined (wc, green) and structured adhered values (sa, purple). Data follow the theory for average $\sigma_{\mathrm{wc}}$ and $\sigma_{\mathrm{sa}}$, respectively. The insets show the same theoretical fluctuation amplitude for the full parameter range, partially inaccessible to experiments. The grey shaded area illustrates the experimentally accessed parameter range.

theory (see Fig. 6). The fluctuations $\left\langle\Delta h^{2}\right\rangle_{\text {distal }}\left(\equiv \psi^{2}\right)$ as a function of membrane tension $\sigma$ are very close to the theoretical curve described by eqn (8) for $\gamma=0$ (Fig. 6a). For the fluctuations at the proximal position as a function of the potential strength $\gamma$, we find convincing agreement between experimental and theoretical data for both adhesion types (Fig. 6b). Here we used the averaged membrane tension, in the weakly confined $\left(\sigma_{\mathrm{wc}}\right)$ and structured adhered $\left(\sigma_{\mathrm{sa}}\right)$ case, though the membrane tension of individual vesicles could differ by more than $50 \%$.

For all vesicles observed, Fig. $6 \mathrm{a}$ and $\mathrm{b}$ show that only part of the decay of the function is probed whereas the plateau region cannot be probed under the experimental conditions used (see insets of Fig. 6). Moreover, all parameters describing the vesicle membrane behaviour near the substrate, i.e., $\psi_{\text {proximal }}$,

Table 3 Comparison of weakly confined and structured adhered systems: mean and standard deviation obtained using DODS ( $N=17$ for the weakly confined system, $N=14$ for the struc. adh. system, unless otherwise stated)

\begin{tabular}{llllr}
\hline System & $\psi_{\text {distal }}[\mathrm{nm}]$ & $\psi_{\text {proximal }}[\mathrm{nm}]$ & $h_{0}[\mathrm{~nm}]$ & $\sigma\left[\mu \mathrm{J} \mathrm{m}^{-2}\right]$ \\
\hline Weakly confined & $67 \pm 19(N=20)$ & $29 \pm 10$ & $605 \pm 428$ & $0.51 \pm 0.37$ \\
Struc. adh. & $82 \pm 23(N=16)$ & $31 \pm 12$ & $402 \pm 252$ & $0.35 \pm 0.25$
\end{tabular}


$\gamma$, and $h_{0}$, exhibit similar values in both systems (see Table 3 ). This indicates that the presence of the substrate influences membrane fluctuations to a similar extent and that even in the structured adhered system the membrane is equilibrated since lipid molecules are free to diffuse over the adhered regions of the vesicle membrane. ${ }^{45}$ In fact, as the substrate preparation within the square fluctuation regions of the structured adhered GUV and the weakly confined GUV was identical, the membrane should encounter similar membrane-substrate interaction if the influence of the pinned membrane sides is negligibly small - and this is observed indeed.

Moreover, in the structured adhered case it appears that the vesicle does not spread further on the patterned substrate. This behaviour is different from previous studies on GUVs adhering strongly to homogeneous substrates - as noticeable by the suppression of fluctuations within the adhesion zone and tension increase. ${ }^{52,84}$ On homogeneous substrates fluctuations bring a new membrane area into contact with the substrate and cause growth of the adhesion zone. In the present case the nonadhesive square areas on the substrate are so large that fluctuations cannot reach to the other side to establish new contacts. Further adhesion is only possible along the adhesive grid, but this involves an increase in bending and vesicle deformation which does not occur. Thus, in the energetics of this system the energy costs due to bending exceed the gain in enthalpy arising from bond formation (and tension, for higher order deformations).

A direct overview of system parameters obtained in the weakly confined and structured adhered case is given in Table 3. Fluctuation amplitudes at the distal membrane yielded a difference of $20 \%$ with slightly larger amplitudes for structured adhered membranes; however, a Wilcoxon rank sum test clarifies the change as not statistically significant (significance level $p=0.12$ ). The same holds for the membrane tension ( $p=0.15)$, but here values for structured adhered membranes were slightly reduced.

Since within fluctuation regions the membrane exhibits the same equilibrium state as in the weakly confined case, the only difference between both systems is the formation of membrane bonds and suppressed fluctuations within adhered regions. ${ }^{28}$ Here, the membrane is flattened and not free to explore all bending modes. Consequently, the membrane excess area within bound regions is smaller than within regions of the free membrane. It is indeed unevenly distributed already between the distal and proximal sides of the GUV as our observation of $\sim 2.5 \times$ enhanced fluctuations at the distal GUV side demonstrates. In this sense the surface excess area is a fundamentally different quantity compared to the membrane tension, which assumes the same value all over the equilibrated fluid lipid membrane. In addition, the vesicle on the structured adhered substrate does not spread, wherefore it maintains its initial surface excess area. Upon adhesion the part of the surface excess area originating from the adhered zone must be redistributed within the GUV and may be shifted from the substrate contact zone to the vesicle hemisphere. We estimated this potential effect on fluctuation amplitudes on the distal side. For a typical vesicle of $15 \mu \mathrm{m}$ radius, pinned over $67 \%$ of the area within the contact zone and by approximating fluctuations as paraboloid dents with $67 \mathrm{~nm}$ amplitude (see Table 3, weakly confined case), their flattening upon adhesion should result in a maximal increase of surface excess area in the vesicle hemisphere by $17 \%$. This larger effective surface area would change the lower integral limit in eqn (8) resulting in fluctuation amplitudes of $81 \mathrm{~nm}$. While such a slight enhancement of fluctuation in the GUV hemisphere upon adhesion is, in principle, possible, the estimated change is yet small enough to lie within the statistical spread of measured amplitudes $(67 \pm 19 \mathrm{~nm}$ for the weakly confined and $82 \pm 23 \mathrm{~nm}$ for the structured adhered GUV). In the present case no significant effect is detectable, but changes in the fluctuation amplitude at the GUV distal site may be probed in more detail with a model system of larger adhesive area and with slowed adhesion kinetics. An interesting extension of our work to further characterize weakly adhering membranes concerns a systematic change of the ratio of non-adhesive/ adhesive areas on the substrate.

\section{Comparison}

Here we have quantified the fluctuation of membranes confined in a weak generic potential using DODS which was crosschecked using RICM wherever possible. When a membrane is close to another surface, fluctuations contribute to the interaction potential. At the same time, fluctuations are a means to measure material properties of the membrane as well as the interaction potential itself. ${ }^{16,30,36,85}$ Hitherto, RICM was the tool of choice for measuring the interaction potential since other techniques, such as flicker spectroscopy, do not give access to the membrane fluctuations near a surface. ${ }^{25,34,86}$ However, the tensions inferred from RICM measurements are usually over-estimated because of the limited resolution in time and in the lateral spatial direction. ${ }^{29,52}$ Furthermore, working solely with fluctuations at the substrate proximal surface, the tension and the potential needs to be determined together in a self-consistent manner. ${ }^{23,87}$

DODS $^{7}$ enabled us to measure at the upper surface of a vesicle to determine the tension independently of the potential, and then use this value to determine the strength of the potential from data gathered at the lower surface of the same vesicle. A key step towards establishing the validity of DODS was its cross-comparison with RICM. This became possible by accounting theoretically for the finite resolution of the two techniques. $^{55}$ We also showed that since our system is in the tension dominated regime, the measurements are highly sensitive to changes in tension, but are not robust against changes in the bending rigidity. For this reason we are not sensitive to complications arising from recent suggestions that the bending rigidity may be different, for example, with small changes in buffer composition. ${ }^{36-38}$ Since small changes are not expected to impact our results, the value available in the literature was used throughout.

In the past, most studies investigated model systems with high ligand numbers, and consequently a state of firm adhesion and tense membrane. ${ }^{30,84,85,88}$ Weak or structured adhesion was 
much less studied. Theoretical work predicts interesting phenomena, for example, geometrically structured/rough substrates result in a decrease of the substrate's attractiveness compared to homogeneous adhesive surfaces and lead to a drop in adhesion energy. ${ }^{89,90}$

We addressed this gap by analysing membrane fluctuations in two different systems - structured adhered or weakly bound, where GUVs were either partially adhered or confined solely by a generic interaction with a substrate. We worked with highly deflated GUVs and therefore, in both cases, the tension primarily acts to reduce thermally-driven membrane undulations. In both cases, the tension was determined to be about $\sigma \sim 0.5 \mu \mathrm{J} \mathrm{m}^{-2}$, the strength of the interaction potential to be $\gamma \sim 4 \mathrm{MJ} \mathrm{m}^{-4}$ - values that are reasonable in light of prior studies. ${ }^{24,29,30,42,80,81,84,85}$ The measured viscosity matched very well with theoretical predictions (see Appendix 1). Similar values for all the measured quantities in the two systems show that in the partially adhered system, though the membrane is selectively bound to the substrate, the nonadhered part of the membrane behaves like its weakly confined counterpart. We can conclude that at least when the tension is low, non-adhered parts of a partly adhered cell or vesicle can be treated independently of the adhered parts.

We found that both our systems are well-described within the Helfrich framework, which then allowed us to measure the strength of the effective interaction potential. In earlier work we showed that this potential is highly non-linear, ${ }^{91}$ and that with decreasing membrane tension, effected via osmotic deflation of GUVs, the minimum of the potential gets further from the substrate and the shape gets broader. ${ }^{45}$ Here we explored the highly deflated case where the membrane is held at the considerable height of $\sim 540 \mathrm{~nm}$, while fluctuating with an amplitude of only $30 \mathrm{~nm}$. These numbers - as well as those reported before - for the membrane height and fluctuation amplitude are difficult to reconcile with the classical idea of extracting the effective potential from a superposition of relevant individual contribution, which typically include gravity, van der Waal's and Helfrich potentials. $^{29,30,52}$ We conclude that the physical origin of the effective potential in eqn (5), is still to be fully understood. However, with the fine description offered by us here and elsewhere, ${ }^{55}$ the measured potential can be used as such as a basis for further studies.

\section{Conclusions}

Two weakly adhered model systems were studied using the techniques DW-RICM and DODS, and the results obtained largely validated the current consensus on membrane dynamics. Let us consider each relevant parameter: at the relatively long wavelengths probed here, the dissipation is expected to come from the viscous damping in the buffer and not from internal membrane friction. Indeed, this is reflected in our experimentally measured values. The membrane tension, $\sigma$, is measured independently of other parameters and is in the expected range. For the bending rigidity the value available in the literature has been used, since our system is in the tension dominated regime and as such is not sensitive to small changes in this parameter.
Our major contribution here has been to shed light on the generic interaction between the substrate and the membrane. The potential is a major player in determining the dynamics of the eventual specific adhesion of the membrane to the substrate, and to our knowledge, this is the first time that the strength of the potential, $\gamma$, was measured from fluctuations, but independently of the tension. Such measurement was realized utilizing the two complementary techniques of DODS - which features high temporal resolution and enabled the decoupling of $\sigma$ and $\gamma$ - and RICM - which features high accuracy in membrane-substrate distance measurements.

In the context of specific ligand-receptor mediated adhesion, the interaction potential plays a dual role - it contributes to defining the membrane-surface distance and its strength, given by the curvature at the minimum, dictates the extent of stochastic exploration by the membrane, ultimately enabling the nucleation of an adhesive patch that may grow. ${ }^{52}$ Fluctuations of weakly adhered systems may be used to probe specific interactions, assessing the change with adhesion bond density, ${ }^{32,42,43}$ for heterogeneous bond distribution, ${ }^{92}$ or during osmolarity changes. ${ }^{45}$ Other potential applications include exploring on-off dynamics of bonds, and weakly confined vesicles subjected to thermal gradients or external shear.

\section{Appendix 1: effective viscosity for a planar membrane surrounded by two different fluids}

The fluctuations of a membrane decay due to the dissipation of energy in the surrounding fluids. In this paragraph, we will calculate the damping coefficients of a membrane surrounded by two different fluids following Seifert. ${ }^{77}$ The membrane is described by the Helfrich Hamiltonian, eqn (5) in the main text. The coordinate system is such that the membrane is on average in the $x y$ plane $(z=0)$ with $z$ perpendicular to the membrane plane. Imposing translational invariance, we reduce the calculation to two dimensions ( $x$ and $z$, without loss of generality). The viscosity of the fluids above and below the membrane are denoted by $\eta^{+}$and $\eta^{-}$, respectively (see Fig. 7). Stokes equations for the hydrodynamics of the fluids around the membrane are

$$
\nabla \cdot \mathbf{v}^{i}=0
$$

$$
\eta^{i} \nabla^{2} \mathbf{v}^{i}=\nabla p^{i}
$$

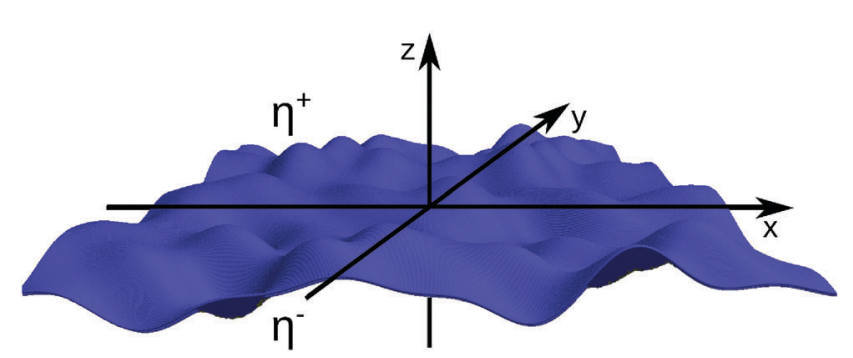

Fig. 7 Sketch of the system introducing the parameters with a membrane at the average position $z=0$ 
with $i=\operatorname{sgn}(z)$. Thereby, $\mathbf{v}^{i}$ is the velocity field $\mathbf{v}^{i} \equiv\left(v_{x}^{i}(x, z), v_{z}^{i}(x, z)\right)$ of the fluids and $p^{i}$ is the pressure field $p^{i}=p^{i}(x, z)$.

The ansatz for the $z$-component of $\mathbf{v}$ imposing a planar incoming wave is given by

$$
v_{z}^{ \pm}=\left[C^{ \pm}+D^{ \pm} q z\right] \mathrm{e}^{\mp q z} \mathrm{e}^{i q x-\Gamma(q) t}
$$

with constraints $\mathbf{v}(z \rightarrow \pm \infty)=\mathbf{0}$. Using eqn (12), we find the $x$-component of the velocity field $v_{x}^{ \pm}$. Moreover, the velocity field has to be continuous at $z=0$ and the in-plane divergence of $v_{x}$ has to vanish at $z=0$, i.e., $\partial_{x} v_{x}(x, z=0)=0$. All together, we find after some algebra $C^{ \pm}= \pm D^{ \pm}$. Now, we calculate the pressure field $p^{ \pm}(x, z)$ with eqn (13) and finally use force balance at $z=0$,

$$
-T_{z z \mid z=0}^{+}+T_{z z \mid z=0}^{-}=-\frac{\delta \mathcal{H}}{\delta h_{\mathbf{q} \mid z=0}}
$$

with the liquid-stress-tensor $T_{k l}^{ \pm}=-p^{ \pm} \delta_{k l}+\eta^{ \pm}\left(\partial_{k} v_{l}^{ \pm}+\partial_{l} v_{k}^{ \pm}\right)$. Combining the results for the pressure fields and the velocity fields, we finally resolve the damping coefficients

$$
\Gamma(q)=\frac{\kappa q^{3}+\sigma q}{2\left(\eta^{+}+\eta^{-}\right)} \equiv \frac{\kappa q^{3}+\sigma q}{4 \eta_{\mathrm{eff}}},
$$

with $\eta_{\text {eff }} \equiv\left(\eta^{+}+\eta^{-}\right) / 2$. Thus, the effective viscosity for a membrane surrounded by different fluids is given by the arithmetic mean of both viscosities.

\section{Appendix 2: spectral boundaries for a quasi-planar membrane}

Over the years, the membrane was described in various approximations. A first approximation was the quasi-spherical geometry for the vesicle shape and, for adhesion to a flat substrate, a quasiplanar membrane was considered. Both representations provide fluctuation amplitudes from theoretical modelling. Here, we will compare both fluctuation amplitudes, in the quasi-spherical and in the quasi-planar geometry, and consequently determine the spectral boundaries in the planar geometry.

In quasi-planar geometry, the fluctuation amplitude for a membrane far away from the substrate (i.e., without the interaction potential term) is known to be

$$
\left\langle\Delta h^{2}\right\rangle=\frac{k_{\mathrm{B}} T}{2 \pi} \int_{q_{\min }}^{q_{\max }} \mathrm{d} q \frac{1}{\kappa q^{3}+\sigma q} .
$$

Therefore, the upper integration limit is given by $q_{\max }=2 \pi / d$ with $d$ being the size of a lipid. The lower integration limit is not known in detail; however, it is of the order of $1 / R$, where $R$ is the radius of the vesicle.

In the following we will derive a more accurate estimate for $q_{\text {min }}$. In quasi-spherical geometry, we expand the membrane shape

$$
R(\Omega, t)=R(1+u(\Omega, t)),
$$

around its mean spherical shape with radius $R$ and fluctuations $u(\Omega, t)$ at the angle $\Omega$. Following the calculation shown in Milner and Safran, ${ }^{13}$ the fluctuation amplitude $\left\langle u^{2}\right\rangle$ in quasi-spherical

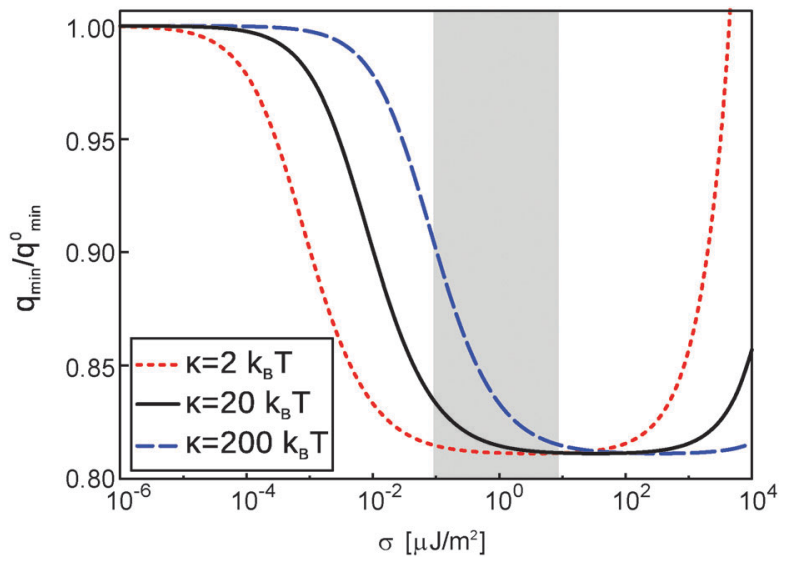

Fig. 8 Lower integration limit $q_{\min }$ as a function of the membrane tension $\sigma$. The vesicle radius was kept constant at $R=10 \mu \mathrm{m}$ while the grey shaded area illustrates the experimentally accessible parameter range.

geometry is given by

$$
\left\langle u^{2}\right\rangle=R^{2} \sum_{l=2}^{l_{\max }}\left\langle\left|u_{l m}\right|^{2}\right\rangle \frac{2 l+1}{4 \pi},
$$

with

$$
\left\langle\left|u_{l m}\right|^{2}\right\rangle=\frac{k_{\mathrm{B}} T}{(l+2)(l-1)\left[\kappa(l+1) l+\sigma R^{2}\right]} .
$$

Therefore, the maximal order of spherical harmonics $l_{\max }$ is the order $R / d$.

The limits for $\sigma \rightarrow 0$ are found in both geometries as

$$
\begin{aligned}
\left\langle\Delta h^{2}\right\rangle_{\sigma \rightarrow 0} & \simeq \frac{k_{\mathrm{B}} T}{4 \pi \kappa} \frac{1}{\left(q_{\min }^{0}\right)^{2}}, \\
\left\langle u^{2}\right\rangle_{\sigma \rightarrow 0} & \simeq \frac{k_{\mathrm{B}} T}{4 \pi \kappa} \frac{R^{2}}{3} .
\end{aligned}
$$

The relative errors due to various approximations is below $1 \%$ for $q_{\max }>10 q_{\min }$ in eqn (21) and below $1 \%$ for $l_{\max } \geq 17$ in eqn (22). Finally, from this we find the correct lower integration limit for the fluctuation amplitude in quasi-planar geometry as

$$
q_{\min }^{0}=\frac{\sqrt{3}}{R}
$$

Including membrane tension, the lower integration limit $q_{\min }$ is found numerically from eqn (17) and (18) and we show $q_{\min }$ in Fig. 8 for typical values of a vesicle $\left(\kappa=2,20,200 k_{\mathrm{B}} T\right.$, and $R=10 \mu \mathrm{m})$. We find $q_{\mathrm{min}}$ to remain close to $q_{\mathrm{min}}^{0}$ for a broad range of membrane tensions. However, for typical membrane tensions of a vesicle we determine $q_{\mathrm{min}} \simeq 0.81 q_{\mathrm{min}}^{0}$. Only for large membrane tensions, $\sigma \gg \kappa / d^{2} \simeq 0.1 \mathrm{~J} \mathrm{~m}^{-2}$, which are far beyond the lysis tension of biomembranes, we find prominent deviations from $q_{\min }^{0}$ which saturate at $q_{\min } \simeq 5.09 q_{\min }^{0}$ for $\sigma \rightarrow \infty$.

\section{Conflict of interest}

The authors declare no competing financial interests. 


\section{Acknowledgements}

C. M. is grateful for the financial support by DAAD and Fonds der Chemischen Industrie. A.-S. S. and D. S. received funding from the European Research Council (starting grant 2013-337283), the Cluster of Excellence: "Engineering of Advanced Materials", and the Research Training Group 1962 of the Friedrich-Alexander Universität Erlangen-Nürnberg. K. S. thanks the European Research Council for funding via grant no. 307104 FP/20072013/ERC/SYNINTER and AMIDEX grant Affinity.

\section{References}

1 W. Helfrich, Elastic properties of lipid bilayers: theory and possible experiments, Z. Naturforsch., C: Biochem., Biophys., Biol., Virol., 1973, 28, 693-703.

2 C. Monzel and K. Sengupta, Measuring shape fluctuations in biological membranes, J. Phys. D: Appl. Physics, 2016, 49, 107791.

3 R. M. Servuss and W. Helfrich, Mutual Adhesion of Lecithin Membranes at Ultralow Tensions, J. Phys., 1989, 50, 809-827.

4 Y. Park, M. Diez-Silva, G. Popescu, G. Lykotrafitis, W. Choi, M. S. Feld and S. Suresh, Refractive index maps and membrane dynamics of human red blood cells parasitized by Plasmodium falciparum, Proc. Natl. Acad. Sci. U. S. A., 2008, 105, 13730-13735.

5 T. Betz, M. Lenz, J.-F. Joanny and C. Sykes, ATP-dependent mechanics of red blood cells, Proc. Natl. Acad. Sci. U. S. A., 2009, 106, 15320-15325.

6 D. Cojoc, S. Finaurini, P. Livshits, E. Gur, A. Shapira, V. Mico and Z. Zalevsky, Toward fast malaria detection by secondary speckle sensing microscopy, Biomed. Opt. Express, 2012, 3, 991-1005.

7 C. Monzel, D. Schmidt, C. Kleusch, D. Kirchenbüchler, U. Seifert, A.-S. Smith, K. Sengupta and R. Merkel, Measuring fast stochastic displacements of bio-membranes with dynamic optical displacement spectroscopy, Nat. Commun., 2015, 6, 8162 .

8 E. A. Evans and V. A. Parsegian, Thermal-mechanical fluctuations enhance repulsion between bimolecular layers, Proc. Natl. Acad. Sci. U. S. A., 1986, 83, 7132-7136.

9 R. Podgornik and V. Parsegian, Thermal-Mechanical Fluctuations of Fluid Membranes in Confined Geometries: The Case of Soft Confinement, Langmuir, 1992, 8, 557-562.

10 L. B. Freund, Entropic pressure between biomembranes in a periodic stack due to thermal fluctuations, Proc. Natl. Acad. Sci. U. S. A., 2013, 110, 2047-2051.

11 E. Evans and W. Rawicz, Entropy-Driven Tension and Bending Elasticity in Condensed-Fluid Membranes, Phys. Rev. Lett., 1990, 64, 2094-2097.

12 J. F. Faucon, M. D. Mitov, P. Méléard, I. Bivas and P. Bothorel, Bending elasticity and thermal fluctuations of lipid membranes. Theoretical and experimental requirements, J. Phys., 1989, 50, 2389-2414.

13 S. T. Milner and S. A. Safran, Dynamical fluctuations of droplet microemulsions and vesicles, Phys. Rev. A: At., Mol., Opt. Phys., 1987, 36, 4371-4379.
14 U. Seifert, Configurations of fluid membranes and vesicles, Adv. Phys., 1997, 46, 13-137.

15 R. Lipowsky and E. Sackmann, Structure and Dynamics of Membranes: I. From Cells to Vesicles/II. Generic and Specific Interactions, North Holland, Amsterdam, 1995.

16 F. Ahmadpoor and P. Sharma, Thermal fluctuations of vesicles and nonlinear curvature elasticity-implications for size-dependent renormalized bending rigidity and vesicle size distribution, Soft Matter, 2016, 12, 2523-2536.

17 U. Seifert and S. A. Langer, Viscous modes of fluid bilayer membranes, Europhys. Lett., 1993, 23, 71-76.

18 E. Evans and A. Yeung, Hidden dynamics in rapid changes of bilayer shape, Chem. Phys. Lipids, 1994, 73, 39-56.

19 E. Evans, Bending resistance and chemically-induced moments in membrane bilayers, Biophys. J., 1974, 14, 923-931.

20 J.-B. Fournier and C. Barbetta, Direct Calculation from the Stress Tensor of the Lateral Surface Tension of Fluctuating Fluid Membranes, Phys. Rev. Lett., 2008, 100, 078103.

21 P. Girard, J. Prost and P. Bassereau, Passive or active fluctuations in membranes containing proteins, Phys. Rev. Lett., 2005, 94, 088102.

22 O. Farago, Membrane fluctuations near a plane rigid surface, Phys. Rev. E: Stat., Nonlinear, Soft Matter Phys., 2008, 78, 051919.

23 T. R. Weikl, M. Asfaw, H. Krobath, B. Rozycki and R. Lipowsky, Adhesion of membranes via receptor-ligand complexes: Domain formation, binding cooperativity, and active processes, Soft Matter, 2009, 5, 13.

24 J. Pécréaux, H.-G. Döbereiner, J. Prost, J.-F. Joanny and P. Bassereau, Refined contour analysis of giant unilamellar vesicles, Eur. Phys. J. E: Soft Matter Biol. Phys., 2004, 13, 277-290.

25 H.-G. Döbereiner, G. Gompper, C. Haluska, D. Kroll, P. Petrov and K. Riske, Advanced Flicker Spectroscopy of Fluid Membranes, Phys. Rev. Lett., 2003, 91, 048301.

26 R. Hirn, T. M. Bayerl, J. O. Rädler and E. Sackmann, Collective membrane motions of high and low amplitude, studied by dynamic light scattering and micro-interferometry, Faraday Discuss., 1998, 111, 17-30.

27 J.-B. Manneville, P. Bassereau, S. Ramaswamy and J. Prost, Active membrane fluctuations studied by micropipet aspiration, Phys. Rev. E: Stat., Nonlinear, Soft Matter Phys., 2001, 64, 021908.

28 C. Monzel, S. Fenz, R. Merkel and K. Sengupta, Probing biomembrane dynamics by dual-wavelength reflection interference contrast microscopy, ChemPhysChem, 2009, 10, 2828-2838.

29 J. O. Rädler, T. J. Feder, H. H. Strey and E. Sackmann, Fluctuation analysis of tension-controlled undulation forces between giant vesicles and solid substrates, Phys. Rev. E: Stat. Phys., Plasmas, Fluids, Relat. Interdiscip. Top., 1995, 51, 4526-4536.

30 R. Bruinsma, A. Behrisch and E. Sackmann, Adhesive switching of membranes: experiment and theory, Phys. Rev. E: Stat. Phys., Plasmas, Fluids, Relat. Interdiscip. Top., 2000, 61, 4253-4267.

31 D. Cuvelier and P. Nassoy, Hidden dynamics of vesicle adhesion induced by specific stickers, Phys. Rev. Lett., 2004, 93, 228101. 
32 A.-S. Smith, K. Sengupta, S. Goennenwein, U. Seifert and E. Sackmann, Force-induced growth of adhesion domains is controlled by receptor mobility, Proc. Natl. Acad. Sci. U. S. A., 2008, 105, 6906-6911.

33 A.-S. Smith, S.-F. Fenz and K. Sengupta, Interferring spatial organization of bonds within adhesion clusters by exploiting fluctuations of soft interfaces, Europhys. Lett., 2010, 89, 28003.

34 F. Brochard-Wyart and J.-F. Lennon, Frequency spectrum of the flicker phenomenon in erythrocyte, J. Phys., 1975, 36, 1035-1047.

35 J. Evans, W. Gratzer, N. Mohandas, K. Parker and J. Sleep, Fluctuations of the red blood cell membrane: relation to mechanical properties and lack of ATP dependence, Biophys. J., 2008, 94, 4134-4144.

36 R. Dimova, Recent developments in the field of bending rigidity measurements on membranes, Adv. Colloid Interface Sci., 2014, 208, 225-234.

37 J. F. Nagle, M. S. Jablin, S. Tristram-Nagle and K. Akabori, What are the true values of the bending modulus of simple lipid bilayers?, Chem. Phys. Lipids, 2015, 185, 3-10.

38 R. M. Venable, F. L. Brown and R. W. Pastor, Mechanical properties of lipid bilayers from molecular dynamics simulation, Chem. Phys. Lipids, 2015, 192, 60-74.

39 C. L. Armstrong, W. Häußler, T. Seydel, J. Katsaras and M. C. Rheinstädter, Nanosecond lipid dynamics in membranes containing cholesterol, Soft Matter, 2014, 10, 2600-2611.

40 M. Arnold, E. A. Cavalcanti-Adam, R. Glass, J. Blümmel, W. Eck, M. Kantlehner, H. Kessler and J. P. Spatz, Activation of integrin function by nanopatterned adhesive interfaces, ChemPhysChem, 2004, 5, 383-388.

41 Z.-H. Huang, G. Massiera, L. Limozin, P. Boullanger, M.-P. Valignat and A. Viallat, Sensitive detection of ultra-weak adhesion states of vesicles by interferometric microscopy, Soft Matter, 2010, 6, 1948.

42 S. F. Fenz, T. Bihr, R. Merkel, U. Seifert, K. Sengupta and A.-S. Smith, Switching from ultraweak to strong Adhesion, Adv. Mater., 2011, 23, 2622-2626.

43 S. F. Fenz, A.-S. Smith, R. Merkel and K. Sengupta, Intermembrane adhesion mediated by mobile linkers: Effect of receptor shortage, Soft Matter, 2011, 7, 952.

44 K. H. Biswas, K. L. Hartman, C.-h. Yu, O. J. Harrison, H. Song, A. W. Smith, W. Y. C. Huang, W.-C. Lin, Z. Guo and A. Padmanabhan, et al., E-cadherin junction formation involves an active kinetic nucleation process, Proc. Natl. Acad. Sci. U. S. A., 2015, 112, 10932-10937.

45 C. Monzel, S. F. Fenz, M. Giesen, R. Merkel and K. Sengupta, Mapping fluctuations in biomembranes adhered to micropatterns, Soft Matter, 2012, 8, 6128.

46 U. Seifert, Self-Consistent Theory of Bound Vesicles, Phys. Rev. Lett., 2002, 74, 5060.

47 K. Mecke, T. Charitat and F. Graner, Fluctuating Lipid Bilayer in an Arbitrary Potential: Theory and Experimental Determination of Bending Rigidity, Langmuir, 2003, 19, 2080.
48 U. Seifert and R. Lipowsky, Adhesion of vesicles, Phys. Rev. A: At., Mol., Opt. Phys., 1990, 42, 4768-4771.

49 E. Sackmann and R. F. Bruinsma, Cell adhesion as wetting transition?, ChemPhysChem, 2002, 3, 262-269.

50 S. Goennenwein, M. Tanaka, B. Hu, L. Moroder and E. Sackmann, Functional Incorporation of Intergins into Solid Supported Membrane on Ultrathin Films of Cellulose: Impact on Adhesion, Biophys. J., 2003, 85, 646-655.

51 G. Bell, M. Dembo and P. Bongrand, Competition between nonspecific repulsion and specific bonding, Biophys. J., 1984, 45, 1051-1064.

52 K. Sengupta and L. Limozin, Adhesion of soft membranes controlled by tension and interfacial polymers, Phys. Rev. Lett., 2010, 104, 088101.

53 M. Kraus and U. Seifert, Relaxation modes of an adhering bilayer membrane, J. Phys. II, 1994, 4, 1117-1134.

54 P.-H. Puech, V. Askovic, P.-G. De Gennes and F. BrochardWyart, Dynamics of Vesicle Adhesion: Spreading Versus Dewetting Coupled to Binder Diffusion, Biophys. Rev. Lett., 2006, 1, 85-95.

55 D. Schmidt, C. Monzel, T. Bihr, R. Merkel, U. Seifert, K. Sengupta and A.-S. Smith, Signature of a Nonharmonic Potential as Revealed from a Consistent Shape and Fluctuation Analysis of an Adherent Membrane, Phys. Rev. X, 2014, 4, 021023.

56 M. Schneider, J. Jenkins and W. Webb, Thermal fluctuations of large quasi-spherical bimolecular phospholipid vesicles, J. Phys., 1984, 45, 1457-1472.

57 N. Kučerka, Y. Liu, N. Chu, H. I. Petrache, S. Tristram-Nagle and J. Nagle, Structure of fully hydrated fluid phase DMPC and DLPC lipid bilayers using X-ray scattering from oriented multilamellar arrays and from unilamellar vesicles, Biophys. J., 2005, 88, 2626-2637.

58 Y. Lyatskaya, Y. Liu, S. Tristram-Nagle, J. Katsaras and J. Nagle, Method for obtaining structure and interactions from oriented lipid bilayers, Phys. Rev. E: Stat. Phys., Plasmas, Fluids, Relat. Interdiscip. Top., 2001, 63, 011907.

59 M. C. Rheinstädter, W. Häußler and T. Salditt, Dispersion Relation of Lipid Membrane Shape Fluctuations by Neutron Spin-Echo Spectrometry, Phys. Rev. Lett., 2006, 97, 048103.

60 B.-A. Brüning, S. Prévost, R. Stehle, R. Steitz, P. Falus, B. Farago and T. Hellweg, Bilayer undulation dynamics in unilamellar phospholipid vesicles: effect of temperature, cholesterol and trehalose, Biochim. Biophys. Acta, 2014, 1838, 2412-2419.

61 L. Limozin and K. Sengupta, Quantitative reflection interference contrast microscopy (RICM) in soft matter and cell adhesion, ChemPhysChem, 2009, 10, 2752-2768.

62 S. Marx, J. Schilling, E. Sackmann and R. Bruinsma, Helfrich Repulsion and Dynamical Phase Separation of Multicomponent Lipid Bilayers, Phys. Rev. Lett., 2002, 88, 138102.

63 A. Zidovska and E. Sackmann, Brownian motion of nucleated cell envelopes impedes adhesion, Phys. Rev. Lett., 2006, 96, 048103.

64 J. Schilling, K. Sengupta, S. Goennenwein, A. R. Bausch and E. Sackmann, Absolute interfacial distance measurements 
by dual-wavelength reflection interference contrast microscopy, Phys. Rev. E: Stat., Nonlinear, Soft Matter Phys., 2004, 69, 021901.

65 S. A. Kim, K. G. Heinze and P. Schwille, Fluorescence correlation spectroscopy in living cells, Nat. Methods, 2007, 4, 963-973.

66 J. Enderlein, I. Gregor, D. Patra, T. Dertinger and U. B. Kaupp, Performance of fluorescence correlation spectroscopy for measuring diffusion and concentration, ChemPhysChem, 2005, 6, 2324-2336.

67 T. Betz and C. Sykes, Time resolved membrane fluctuation spectroscopy, Soft Matter, 2012, 8, 5317.

68 A.-S. Smith, B. G. Lorz, U. Seifert and E. Sackmann, Antagonist-induced deadhesion of specifically adhered vesicles, Biophys. J., 2006, 90, 1064-1080.

69 R. R. Netz, Complete Unbinding of Fluid Membranes in the Presence of short-ranged forces, Phys. Rev. E: Stat. Phys., Plasmas, Fluids, Relat. Interdiscip. Top., 1995, 51, 2286-2294.

70 R. Lipowsky and B. Zielinska, Binding and unbinding of lipid membranes: A Monte Carlo study, Phys. Rev. Lett., 1989, 62, 1572-1575.

71 A. Kumar and G. M. Whitesides, Features of gold having micrometer to centimeter dimensions can be formed through a combination of stamping with an elastomeric stamp and an alkanethiol ink followed by chemical etching, Appl. Phys. Lett., 1993, 63, 2002-2004.

72 A. P. Quist, E. Pavlovic and S. Oscarsson, Recent advances in microcontact printing, Anal. Bioanal. Chem., 2005, 381, 591-600.

73 D. S. Dimitrov and M. I. Angelova, Lipid swelling and liposome formation mediated by electric fields, Bioelectrochem. Bioenerg., 1988, 19, 323-336.

74 E. P. Petrov and P. Schwille, Chapter State of the Art and Novel Trends in Fluorescence Correlation Spectroscopy, in Springer Series on Fluorescence, ed. U. Resch-Genger, Springer, New York, 2008, vol. 6, pp. 145-197.

75 J. S. Ploem, Mononuclear Phagocytes in Immunity, Infection and Pathology, Blackwell Scientific, Oxford, 1975.

76 L. Limozin and K. Sengupta, Modulation of vesicle adhesion and spreading kinetics by hyaluronan cushions, Biophys. J., 2007, 93, 3300-3313.

77 U. Seifert, Dynamics of a bound membrane, Phys. Rev. E: Stat. Phys., Plasmas, Fluids, Relat. Interdiscip. Top., 1994, 46, 3124-3127.

78 Y. Zhou and R. M. Raphael, Effect of salicylate on the elasticity, bending stiffness, and strength of SOPC membranes, Biophys. J., 2005, 89, 1789-1801.

79 J. Humpolíčková, E. Gielen, A. Benda, V. Fagulova, J. Vercammen, M. VandeVen, M. Hof, M. Ameloot and
Y. Engelborghs, Probing diffusion laws within cellular membranes by Z-scan fluorescence correlation spectroscopy, Biophys. J., 2006, 91, L23-L25.

80 W. Häckl, U. Seifert and E. Sackmann, Effects of Fully and Partially Solubilized Amphiphiles on Bilayer Bending Stiffness and Temperature Dependence of the Effective Tension of Giant Vesicles, J. Phys. II, 1997, 7, 1141-1157.

81 H. Engelhardt, H. Duwe and E. Sackmann, Bilayer bending elasticity measured by Fourier analysis of thermally excited surface undulations of flaccid vesicles, J. Phys., Lett., 1985, 46, 395-400.

82 S. Cha, S. H. Kim and D. Kim, Viscosity of Sucrose Aqueous Solutions Measured by Using Fluorescence Correlation Spectroscopy, J. Korean Phys. Soc., 2010, 56, 1315-1318.

83 M. Mell, L. H. Moleiro, Y. Hertle, I. López-Montero, F. J. Cao, P. Fouquet, T. Hellweg and F. Monroy, Fluctuation dynamics of bilayer vesicles with intermonolayer sliding: experiment and theory, Chem. Phys. Lipids, 2015, 185, 61-77.

84 A. Albersdörfer, T. Feder and E. Sackmann, Adhesioninduced domain formation by interplay of long-range repulsion and short-range attraction force: A model membrane study, Biophys. J., 1997, 73, 245-257.

85 E. Sackmann and A.-S. Smith, Physics of cell adhesion: some lessons from cell-mimetic systems, Soft Matter, 2014, 10, 1644-1659.

86 K. Fricke, K. Wirthensohn, R. Laxhuber and E. Sackmann, Flicker spectroscopy of erythrocytes. A sensitive method to study subtle changes of membrane bending stiffness, Eur. Biophys. J., 1986, 14, 67-81.

87 A.-S. Smith and U. Seifert, Vesicles as a model for controlled (de-)adhesion of cells: a thermodynamic approach, Soft Matter, 2007, 3, 275-289.

88 S. F. Fenz, R. Merkel and K. Sengupta, Diffusion and intermembrane distance: case study of avidin and E-cadherin mediated adhesion, Langmuir, 2009, 25, 1074-1085.

89 P. S. Swain and D. Andelman, The Influence of Substrate Structure on Membrane Adhesion, Langmuir, 1999, 15, 8902-8914.

90 P. S. Swain and D. Andelman, Supported membranes on chemically structured and rough surfaces, Phys. Rev. E: Stat., Nonlinear, Soft Matter Phys., 2001, 63, 051911.

91 T. Bihr, S. Fenz, E. Sackmann, R. Merkel, U. Seifert, K. Sengupta and A.-S. Smith, Association rates of membrane-coupled cell adhesion molecules, Biophys. J., 2014, 107, L33-L36.

92 V. D. Gordon, T. J. O'Halloran and O. Shindell, Membrane adhesion and the formation of heterogeneities: biology, biophysics, and biotechnology, Phys. Chem. Chem. Phys., 2015, 17, 15522-15533. 